\title{
Transmission properties of hollow-core photonic bandgap fibers in relation to molecular spectroscopy
}

\author{
Falk, Charlotte ljeoma; Hald, Jan; Petersen, Jan C.; Lyngsøe, Jens Kristian
}

Published in:

Applied Optics

Publication date:

2010

Document Version

Publisher's PDF, also known as Version of record

Link back to DTU Orbit

Citation (APA):

Falk, C. I., Hald, J., Petersen, J. C., \& Lyngsøe, J. K. (2010). Transmission properties of hollow-core photonic bandgap fibers in relation to molecular spectroscopy. Applied Optics, 49(49), 3854-3859.

http://www.opticsinfobase.org/view article.cfm?gotourl=http\%3A\%2F\%2Fwww\%2Eopticsinfobase\%2Eorg\%2FDi rectPDFAccess\%2F3C4DBFA6\%2DBDB9\%2D137E\%2DCB21F0557403AE31\%5F203564\%2Epdf\%3Fda\%3D1 \%26id\%3D203564\%26seq\%3D0\%26mobile\%3Dno\&org=Riso\%20National\%20Laboratory\%20AFD\%20Informat ionsservice\%20\%28DNL\%29

\section{General rights}

Copyright and moral rights for the publications made accessible in the public portal are retained by the authors and/or other copyright owners and it is a condition of accessing publications that users recognise and abide by the legal requirements associated with these rights.

- Users may download and print one copy of any publication from the public portal for the purpose of private study or research.

- You may not further distribute the material or use it for any profit-making activity or commercial gain

- You may freely distribute the URL identifying the publication in the public portal 


\title{
Transmission properties of hollow-core photonic bandgap fibers in relation to molecular spectroscopy
}

\author{
Charlotte I. Falk, ${ }^{1, \star}$ Jan Hald, ${ }^{1}$ Jan C. Petersen, ${ }^{1}$ and Jens K. Lyngs $\varnothing^{2}$ \\ 'Danish Fundamental Metrology, Matematiktorvet 307, DK-2800 Kgs. Lyngby, Denmark \\ ${ }^{2}$ NKT Photonics A/S, Blokken 84, DK-3460 Birkerød, Denmark \\ *Corresponding author: cif@dfm.dtu.dk
}

Received 23 April 2010; revised 10 June 2010; accepted 10 June 2010;

posted 11 June 2010 (Doc. ID 127425); published 2 July 2010

\begin{abstract}
The transmission properties of five types of hollow-core photonic bandgap fibers (HC-PBFs) are characterized in the telecom wavelength range around $1.5 \mu \mathrm{m}$. The variations in optical transmission are measured as a function of laser frequency over a $2 \mathrm{GHz}$ scan range as well as a function of time over several hours. The influence of these variations on spectroscopy of molecules in a HC-PBF is simulated. (C) 2010 Optical Society of America

OCIS codes: $\quad 060.2270,060.2370,060.2400,060.5295$.
\end{abstract}

\section{Introduction}

Gas-filled hollow-core photonic bandgap fibers (HCPBFs) can be used for efficient interaction between light and molecules or atoms over long optical path lengths. The tight confinement of light within the HC-PBFs allows for nonlinear interactions at power levels much lower than required in free space [1-3]. Long gas-filled HC-PBFs in connection with tunable or broadband light sources have been proposed as sensitive gas sensors [4-9]. The concentration of the gas of interest is derived from the measured area of an isolated absorption line. High sensitivity in a compact setup is possible with a long coiled HCPBF. However, as the length of the HC-PBF is increased, the response time of the sensor increases as well. For gas entering the HC-PBF by diffusion from the fiber ends, the response time scales with the square of the fiber length [10]. Faster response is possible if small radial holes are laser drilled into the core along the fiber [11] or if the fiber is sectioned with small air gaps between each section [12]. Gasfilled HC-PBFs can also be used as temperature sen-

0003-6935/10/203854-06\$15.00/0

(C) 2010 Optical Society of America sors when the fibers are filled with low-pressure gas. The linewidths of isolated absorption lines are mainly due to the Doppler broadening and, hence, depend on the gas temperature $[13,14]$.

These sensor applications require a well-defined spectroscopic baseline in order to accurately derive the integrated area or the linewidth of an isolated absorption line. The baseline is obtained from the optical transmission of the HC-PBF in the absence of an absorbing gas. Ideally, the baseline should be flat over a frequency range corresponding to a few times the linewidth of the relevant absorption line. Alternatively, if the application allows the fiber to be repeatedly filled and vented, it will be sufficient for the baseline to remain unchanged during the filling time. This will allow the actual baseline to be measured either before or after filling the fiber with gas. The limitation in the accuracy and sensitivity of gas sensors and temperature sensors based on gas-filled $\mathrm{HC}-\mathrm{PBF}$ s due to variations in the spectroscopic baseline with frequency and time is investigated in this paper.

The optical transmissions of five types of HC-PBFs in the telecom wavelength range around $1.5 \mu \mathrm{m}$ are measured as a function of time and as a function of laser frequency over a scan range of about $2 \mathrm{GHz}$. 
Polarization properties of the HC-PBFs are characterized. Simulated absorption profiles are added to these transmission data, which constitute our baselines, and the accuracy at which the integrated areas and linewidths can be recovered by least-squares fitting is determined. Similar measurements and analyses of a standard single-mode optical fiber are made for comparison.

\section{Experimental Configuration}

The experimental setup is shown in Fig. 1. A laser spectrometer developed for accurate measurements of the ${ }^{13} \mathrm{C}_{2} \mathrm{H}_{2} P(16)$ transition at $1542.3837 \mathrm{~nm}$ is used as the light source. The spectrometer is based on two Koheras E15 fiber lasers with a nominal output of $25 \mathrm{~mW}$ : a master laser and a slave laser. The master laser is locked to the acetylene $P(16)$ transition using wavelength modulated saturation spectroscopy in a setup similar to the setup of Balling et al. [15]. The Allan deviation describing the stability of the master laser is below $5 \mathrm{kHz}$ for averaging times between $50 \mathrm{~ms}$ and several hours. The slave laser is offset locked to the master laser using a setup based on the ideas of Schünemann et al. [16]. The offset frequency is set by a Rohde \& Schwartz SML01 signal generator, which is controlled by a personal computer (PC). The frequency of the slave laser is stepped with the signal generator across a $2 \mathrm{GHz}$ frequency range with a dead time of about $50 \mathrm{~ms}$ per frequency step. At each step, the frequency is changed by $20 \mathrm{MHz}$ and the actual differences between the master and the slave laser frequencies, as well as the power transmitted through the HC-PBF under test, are measured for about $50 \mathrm{~ms}$. The difference between the master and the slave laser frequencies deviates less than $10 \mathrm{kHz}$ from the set frequency. Inaccuracies in laser frequencies can be neglected in the following analyses.

The output of the slave laser passes through a fiber-coupled acousto-optic modulator (AOM) and is subsequently collimated in free space. The beam

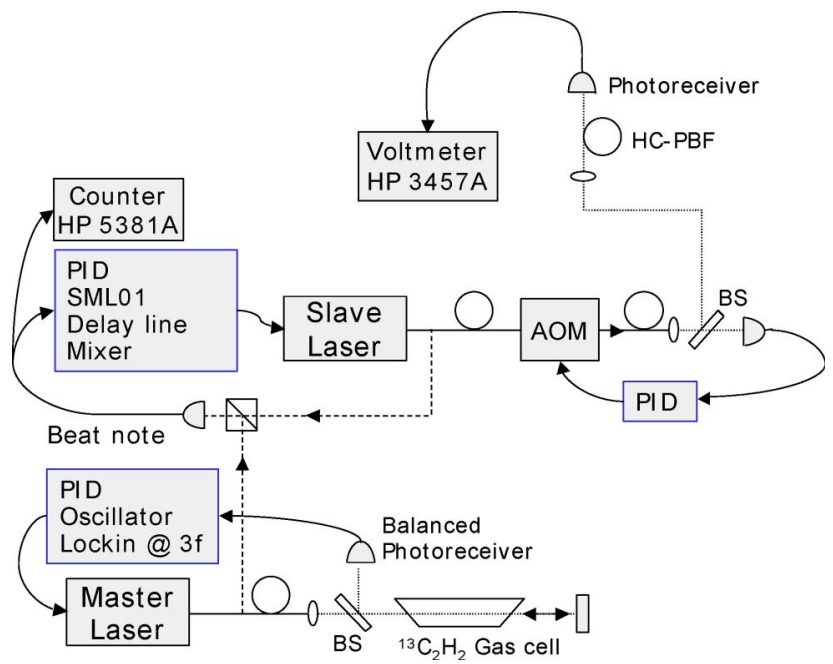

Fig. 1. (Color online) Experimental setup. PID, feedback controller; BS, beam splitter; AOM, acousto-optic modulator. passes an uncoated wedge acting as a beam splitter. The transmitted part goes to a photodetector (New Focus 2033), which is used to stabilize the optical power via feedback to the $\mathrm{RF}$ power driving the AOM. The beam reflected from the beam splitter is carefully mode matched to the HC-PBF under the assumption that core eccentricities are negligible. Two mirrors are used to adjust the beam path, and the $\mathrm{HC}-\mathrm{PBF}$ is mounted on a three-dimensional translational stage. An aspheric lens with a focal length of $8 \mathrm{~mm}$ is placed in front of the HC-PBF for focusing into the fiber. For each fiber, an additional lens is placed in the beam path to improve the coupling efficiency. Various focal lengths and positions for the additional lens are tested while optimizing the position of the $8 \mathrm{~mm}$ lens in order to maximize the coupling efficiency. This additional lens is left out of the setup for those fibers where it does not improve the coupling efficiency.

The HC-PBF under test is coiled with a diameter of about $7 \mathrm{~cm}$ and carefully fixed to an aluminum plate. The ends of the fiber are open and exposed to the air in the laboratory. The output of the fiber is collimated and the optical power, which is typically between 50 and $100 \mu \mathrm{W}$, is detected by using an aspheric lens with a focal length of $4.5 \mathrm{~mm}$ and another New Focus 2033 photoreceiver. The voltage from the photoreceiver is measured with a HP $3457 \mathrm{~A}$ voltmeter. The focusing lens, the HC-PBF, and the photo receiver are placed inside a foam enclosure to minimize turbulence, temperature fluctuations, and stray light. The temperature of the aluminum plate is monitored with a Pt-100 temperature sensor.

The windows in front of the photodiodes have been removed for both New Focus 2033 detectors in order to reduce interference effects. The relative out-of-loop power stability in the absence of the HC-PBF, but with every thing else in place (including lenses), has an Allan deviation below $10^{-5}$ for averaging times between 25 and $2000 \mathrm{~s}$. The relative variations in the detected optical power over the $2 \mathrm{GHz}$ frequency scan are below $0.03 \%$.

Before each set of measurements, both ends of the $\mathrm{HC}-\mathrm{PBF}$ are cleaved and the coupling into the fiber is optimized. Each set of measurements is started during the afternoon, and a PC controls the scanning of the laser frequency and collects data from the HP $3457 \mathrm{~A}$ voltmeter, the temperature sensor, and the laser offset frequency from a HP 5381A counter. Each $2 \mathrm{GHz}$ scan takes about $15 \mathrm{~s}$. The measurement continues overnight and the data collected between 10 p.m. and 6:30 a.m., when the laboratory environment is most stable, are used.

Four sets of measurements are completed for each type of HC-PBF. The tested HC-PBF types are listed in Table 1 together with their specified and measured properties. All five HC-PBFs were manufactured by NKT Photonics and cut to a length of $3.0 \mathrm{~m}$. The first three HC-PBFs are HC19-1550-01, HC1550-02, and HC1550-PM-01 [17]. HC-PBF 4 is similar to HC-PBF 3, but with an outer diameter 
scaled down by approximately $2 \%$, so that $1550 \mathrm{~nm}$ is located $30 \mathrm{~nm}$ closer to the long wavelength edge of the fiber's low loss region. HC-PBF 5 is similar to HC-PBF 2, but with a cladding air-filling fraction of $84 \%$. The cladding pitch and core size were scaled accordingly to maintain the bandgap at $1550 \mathrm{~nm}$.

The same measurements are performed for a $1 \mathrm{~m}$ long standard SMF-28 fiber with FC/APC connectors, which is used as a reference. The coupling efficiencies listed in Table $\underline{1}$ are determined as the measured transmission corrected for the bulk loss specified in Table 1 . The bulk loss was determined at NKT Photonics using the cutback method. The coupling efficiency uncertainties are calculated as the standard deviation over the measurements from each realignment of the coupling into a given fiber.

\section{Polarization Properties}

Two of the five HC-PBFs in this study are manufactured as polarization-maintaining fibers. The polarization properties of these fibers are determined by measuring the four Stokes parameters of the HCPBF output for different orientations of the linearly polarized input field. The Stokes parameters are given by

$$
\begin{aligned}
& S_{0}=P_{H}+P_{V}, \quad S_{1}=P_{H}-P_{V}, \\
& S_{2}=P_{+45}-P_{-45}, \quad S_{3}=P_{\sigma^{+}}-P_{\sigma^{-}},
\end{aligned}
$$

where $P_{H}, P_{V}, P_{+45}, P_{-45}, P_{\sigma^{+}}$, and $P_{\sigma^{-}}$denote the power of the horizontally polarized light, the vertically polarized light, the light polarized +45 degrees to the horizontal plane, the light polarized -45 degrees to the horizontal plane, the right-hand circularly polarized light and the left-hand circularly polarized light, respectively. The degree of polarization is defined as [18]

$$
V=\frac{\sqrt{S_{1}^{2}+S_{2}^{2}+S_{3}^{2}}}{S_{0}} .
$$

The Stokes parameters are measured using the following procedure: a quarter-wave plate and a half- wave plate are calibrated so that they each rotate a linearly polarized beam in such a way that an equal amount of the beam is transmitted through and reflected from a polarizer placed after the wave plate. A polarizer is placed at the output end of the fiber and the power of the transmitted and reflected beams are measured in the three cases: without any wave plate at the output end, with the calibrated quarter-wave plate placed between the fiber output end and the polarizer, and, third, with the calibrated quarter-wave plate replaced by the calibrated half-wave plate. These measurements are repeated for various orientations of the input beam polarization. The maximum and minimum degree of polarization for each of the two polarization-maintaining fibers and for fiber HC-PBF 5 are listed in Table 1 . We find that HC-PBF 5 behaves as a polarization-maintaining fiber even though it was not intentionally manufactured as such. The minimum degree of polarization in Table 1 is well below $100 \%$, which shows that the HC-PBF can transform perfectly polarized light into partially polarized light. This is possible if the HC-PBF output is a superposition of multiple free-space spatial modes having different polarizations.

The fibers HC-PBF 1 and HC-PBF 2 did not maintain a stable output polarization during the time needed to characterize the polarization properties.

\section{Analysis of Transmission Data}

The transmission of the fiber, i.e. the ratio between the output power of the fiber and the input power, is measured as the laser frequency is scanned across the $2 \mathrm{GHz}$ range. The transmission measurements are subsequently normalized to the mean transmission for each scan. The variation in transmission listed in Table 1 is the difference between the maximum transmission and the minimum transmission for a single scan. This variation over the $2 \mathrm{GHz}$ scan ranges from $0.30 \%$ to $3.59 \%$ for the HC-PBFs and $0.02 \%$ to $0.10 \%$ for the SMF-28. Figure 2(a) shows the normalized transmission with the smallest variation for each fiber, while Fig. 2(b) shows the normalized transmission with the largest variation. Figure $\underline{2}$ shows that the HC-PBFs have nonflat

Table 1. Specified Parameters (Rows 1-5) and Measured Parameters (Rows 6-9) for Each of the Tested Fibers ${ }^{a}$

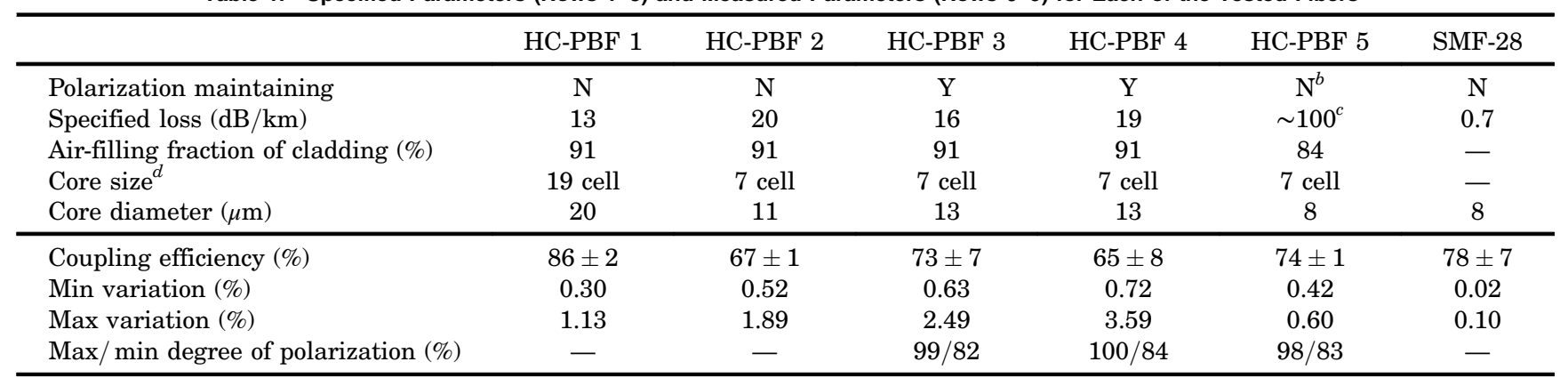

${ }^{a}$ Further details of the parameters are given in the text.

${ }^{b}$ The $3 \mathrm{~m}$ of fiber used in these experiments behaved as a polarization-maintaining fiber, though it was not manufactured as such.

${ }^{c}$ Estimated by NKT Photonics.

${ }^{d}$ In units of cladding cells. 

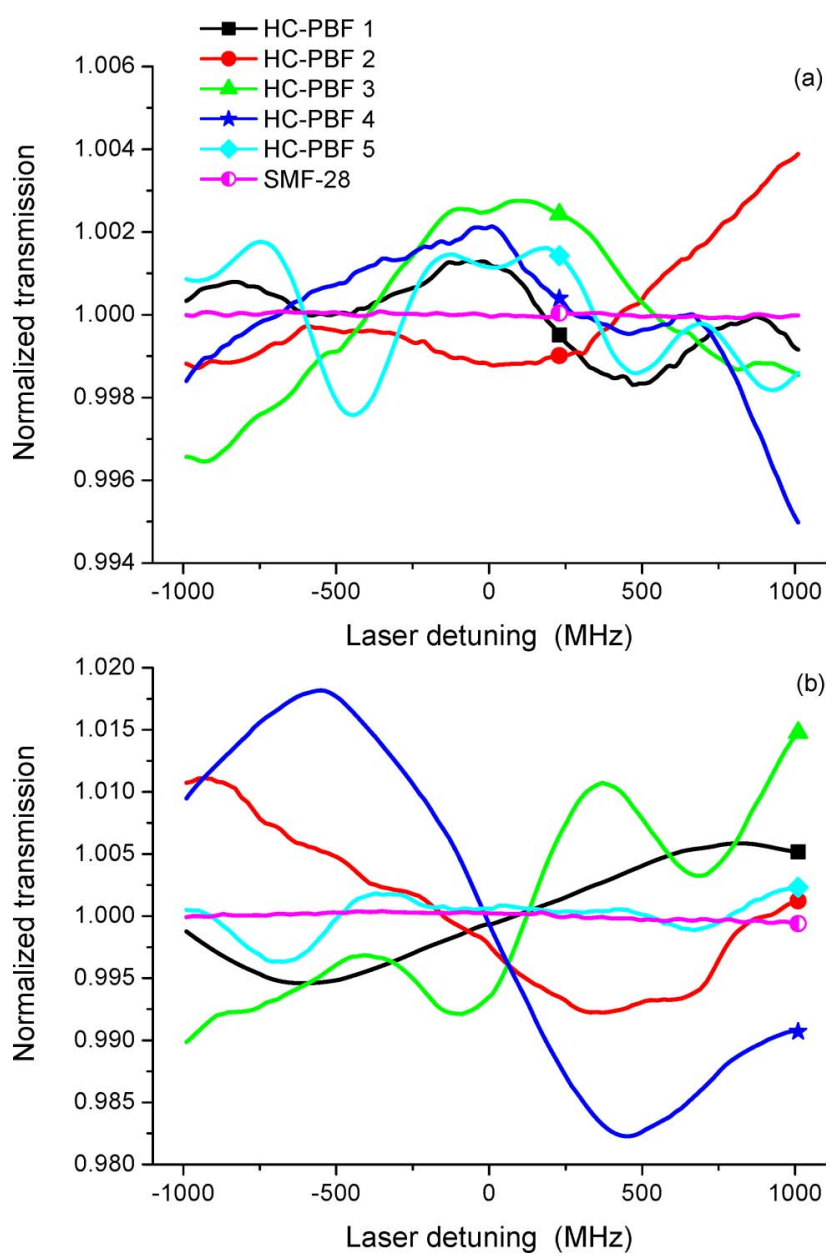

Fig. 2. (Color online) Normalized transmission through the fibers as the laser frequency is scanned over $2 \mathrm{GHz}$ (a) for the measurements with the smallest variation for each fiber type and (b) for the measurements with the largest variation. Note that the scalings of the vertical axes are not identical.

transmission spectra and that the spectra change over time. This change is not only due to different in-coupling conditions, but it has also been observed to occur over time within each set of measurements. A comparison between the coupling efficiencies and the transmission variations listed in Table 1 shows that the HC-PBFs with the largest variation in transmission are the ones with the lowest coupling efficiencies. Lower coupling efficiency could imply a higher degree of coupling to surface modes or higher-order core modes. HC-PBF 1 has the largest core size and would, therefore, be expected to support a larger number of higher-order modes, which, in turn, could lead to a higher variation in transmission. However, a larger core is also expected to reduce the number of surface modes [19]. Interference between coupled core modes and surface modes is known to give significant baseline variations in the output from HC-PBFs [12].

Averaging over time can reduce the variance of the transmission and, thus, increase the sensitivity of a sensor based on a gas-filled HC-PBF. The Allan var- iance method was introduced to analyze the stability over time for frequency standards [20,21], but the method has also been used to characterize gas sensors based on laser spectroscopy $[22,23]$. The Allan variance of the transmission at a certain laser frequency is the average of the squared differences between successive values of the transmission measured with a specified averaging time. For a set of successive transmission measurements comprising $N$ data points $\left(T_{i}, i=1, \cdots, N\right)$ at a certain laser frequency, the Allan variance corresponding to the averaging time $\tau$ is given by

$$
\left\langle\sigma_{A}^{2}(s)\right\rangle_{\tau}=\frac{1}{2(m-1)} \sum_{k=1}^{m-1}\left[A_{k+1}(s)-A_{k}(s)\right]^{2},
$$

where

$$
A_{k}(s)=\frac{1}{s} \sum_{i=1+s(k-1)}^{s k} T_{i}, \quad k=1, \cdots, m .
$$

$m$ is the largest integer less than or equal to $N / s$, and $s$ is the number of data points sampled during the time $\tau$. The Allan deviation, which is the square root of the Allan variance, is for a given set of measurements calculated for each laser frequency using all data from 10 p.m. to 6:30 a.m. The normalized transmission Allan deviation is calculated as the average over the Allan deviations for each laser frequency in the $2 \mathrm{GHz}$ scan range. A normalized transmission Allan deviation is obtained from each set of measurements, and we obtain four normalized transmission Allan deviation graphs for each type of fiber. Figure 3 shows the normalized transmission Allan deviations with the smallest minimum value for each type of fiber. The optimum averaging time for the five HCPBFs is between 100 and $200 \mathrm{~s}$, while the optimum averaging time for the SMF-28 is above $2000 \mathrm{~s}$. The minimum normalized transmission Allan deviation for the HC-PBFs lies between $2 \times 10^{-5}$ and $6 \times 10^{-5}$. The various normalized transmission Allan

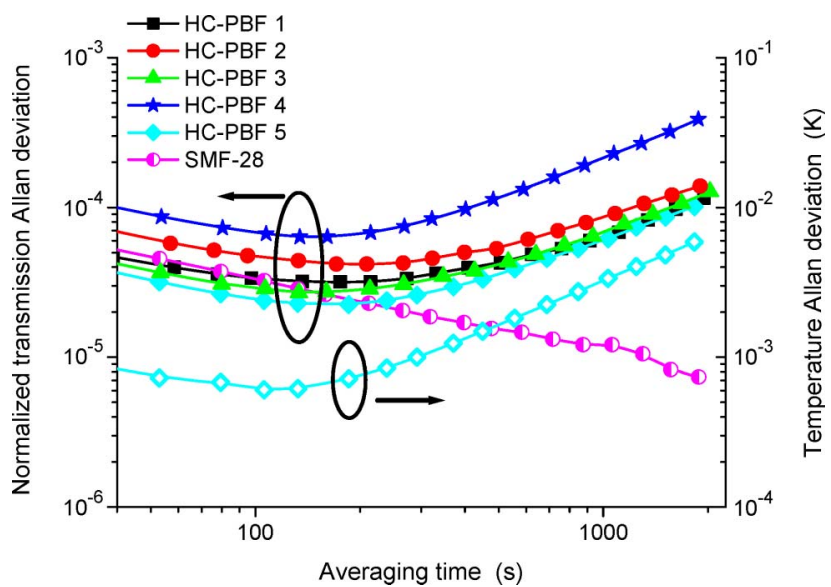

Fig. 3. (Color online) Normalized transmission Allan deviation with the smallest minimum for each fiber. The open diamond symbols represent the temperature Allan deviation corresponding to the plotted HC-PBF 5 transmission data. 
deviations obtained for each fiber show some variations, but they all follow the same general trend for a given type of fiber. The Allan deviation for the temperature measurements corresponding to the normalized transmission Allan deviation is plotted for HC-PBF 5 in Fig. 3. This temperature Allan deviation has a shape similar to the normalized transmission Allan deviations for the hollow-core fibers. In particular, they all have a minimum between 100 and $200 \mathrm{~s}$. This indicates that the temporal variations in the hollow-core fiber transmissions are coupled to temperature variations, which was also observed in [12]. The interference between different modes in a fiber depends on the temperature [24]. This could explain why the transmission Allan deviation for the SMF-28 differs from the hollow-core fibers. Since the SMF-28 supports only the fundamental mode, there are no higher-order modes or surface modes to exhibit temperature-dependent interference.

\section{Simulated Absorption Spectra}

The measured transmission spectra at 10 p.m., 12 a.m., 2 a.m., 4 a.m., and 6 a.m. from each set of measurements are multiplied by the mathematical expression for the optical transmission near an isolated Doppler broadened molecular absorption line given by

$$
T(\Delta)=\exp \left(-\frac{\alpha}{\sqrt{\pi \delta \nu_{D}^{2}}} e^{-\Delta^{2}} / \delta \nu_{D}^{2}\right)
$$

where $\Delta$ is the laser detuning, $\alpha$ is the area of the absorption line, which is set to $0.5 \cdot \pi^{1 / 2} \delta \nu_{D}$, and $\delta \nu_{D}$ is the $1 / e$ Doppler width, which is set to $260 \mathrm{MHz}$ for a typical light molecule at room temperature with absorption lines in the wavelength range around $1550 \mathrm{~nm}$.

For each modeled spectrum, the linewidth and the area of the absorption line are retrieved using a least-squares fitting routine. The relative differences between the model area $\left(0.5 \cdot \pi^{1 / 2} \delta \nu_{D}\right)$ and the retrieved areas are plotted in Fig. 4(a). The numbers listed below the measurement data in Fig. 4 are the standard deviation calculated over the 20 data points for the corresponding fiber.

In a modified analysis, the modeled spectra are normalized with the fiber transmission measured 30 min later. This corresponds to the situation where the fiber is evacuated in $30 \mathrm{~min}$ and a subsequent measurement of the transmission is used as the baseline. The $30 \mathrm{~min}$ are chosen as a typical time needed for filling or venting a $3 \mathrm{~m}$ long $\mathrm{HC}-\mathrm{PBF}$ [10]. Figure 4(b) shows the relative differences between the model area and the retrieved areas in this case. The relative differences are clearly reduced by this normalization.

The relative errors in the retrieved linewidths follow, in general, the same trend as the relative error in the retrieved areas. The largest standard deviation for the relative linewidth errors is obtained
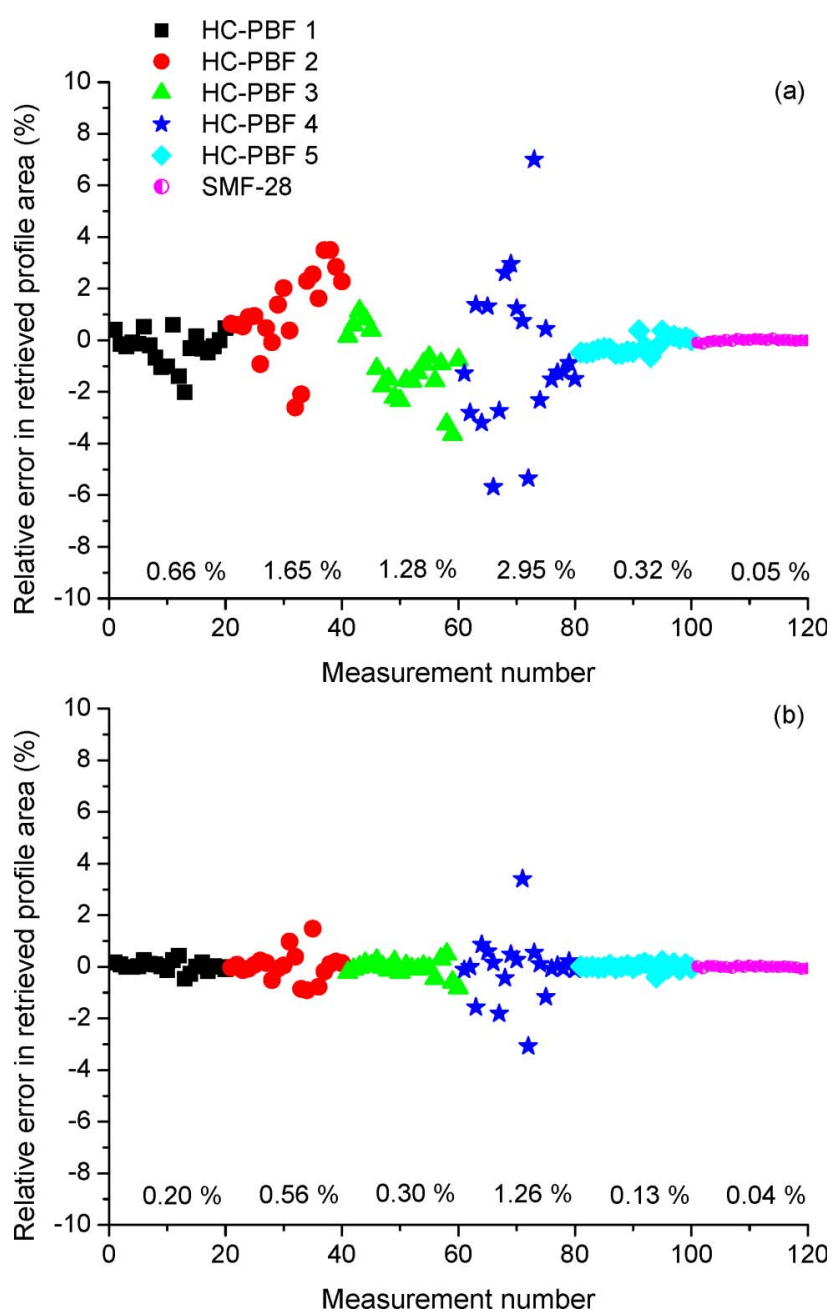

Fig. 4. (Color online) (a) Relative errors in the retrieved absorption line area for each of the tested fibers when modeled with a Gaussian profile. The numbers listed below the measurement data are the standard deviations calculated over the 20 data points for the corresponding fiber. (b) Similar to (a), but with each modeled spectrum normalized to the measured fiber transmission $30 \mathrm{~min}$ later.

for HC-PBF 4 (1.6\%), whereas HC-PBF 5 gives the smallest standard deviation for the relative linewidth errors $(0.28 \%)$. These standard deviations can be reduced by about a factor of 2 if the modeled spectra are normalized to the transmission measured $30 \mathrm{~min}$ later. The relative errors in the retrieved linewidths will induce a 2 times larger relative error in the temperature reading of a thermometer based on molecular spectroscopy in a HC-PBF since the linewidth scales with the square root of the temperature. For the best fiber at room temperature, these data correspond to a typical uncertainty of $2 \mathrm{~K}$ in a configuration without cycling of the gas pressure.

If the Gaussian line shape is replaced with a Lorentzian line shape of the same FWHM linewidth, which is representative for the collisional broadening regime, the standard deviation of the relative errors in area and linewidth increases with a factor of about 2 . 


\section{Conclusions}

The transmission properties of five different HCPBFs have been characterized as a function of laser frequency over a scan range of $2 \mathrm{GHz}$ and as a function of time over several hours. The influence of the variations in transmission on the potential accuracy of sensors based on gas-filled HC-PBFs has been determined. For short measurement times, where the baseline does not change (i.e., a few minutes or shorter), the baseline variations contribute to the overall gas sensor uncertainty by $0.3 \%$ to $3.0 \%$, depending on the actual HC-PBF. This can be improved by a factor of 2 to 4 if the gas pressure is cycled and the baseline is measured with the empty fiber. The typical uncertainty for a thermometer based on molecular spectroscopy in a $\mathrm{HC}-\mathrm{PBF}$ is found to be about $2 \mathrm{~K}$ at room temperature.

The temporal variation of the HC-PBF transmission is found to be correlated to the variation in temperature. The temperature Allan deviation for $30 \mathrm{~min}$ of averaging time is about $5 \mathrm{mK}$. Reduction in temperature variation by active stabilization is expected to improve the temporal variation of the transmission. Alternatively, mechanical vibration of the HC$\mathrm{PBF}$, as well as rapid variations in $\mathrm{HC}-\mathrm{PBF}$ temperature, can be used to average out the transmission variations [12].

The fiber $\overline{H C}$-PBF 5 , which is expected to have the largest suppression of higher-order modes, has the smallest variations in optical transmission over the $2 \mathrm{GHz}$ scan range, as well as over time.

This work was financially supported by the European Community's 7th Framework Programme, ERA-NET+, under the iMERA + project-grant agreement 217257.

\section{References}

1. R. Thapa, K. Knabe, M. Faheem, A. Naweed, O. L. Weaver, and K. L. Corwin, "Saturated absorption spectroscopy of acetylene gas inside large-core photonic bandgap fiber," Opt. Lett. 31, 2489-2491 (2006).

2. J. Hald, J. C. Petersen, and J. Henningsen, "Saturated optical absorption by slow molecules in hollow-core photonic bandgap fibers," Phys. Rev. Lett. 98, 213902 (2007).

3. P. Londero, V. Venkataraman, A. R. Bhagwat, A. D. Slepkov, and A. L. Gaeta, "Ultralow-power four-wave mixing with Rb in a hollow-core photonic band-gap fiber," Phys. Rev. Lett. 103, 043602 (2009).

4. T. Ritari, J. Tuominen, H. Ludvigsen, J. C. Petersen, T. Sørensen, T. P. Hansen, and H. R. Simonsen, "Gas sensing using air-guiding photonic bandgap fibers," Opt. Express 12, 4080-4087 (2004).

5. A. Lambrecht, S. Hartwig, J. Herbst, and J. Wöllenstein, "Hollow fibers for compact infrared gas sensors," Proc. SPIE 6901, 69010V (2008).
6. X. Zhou, J. Hou, and J. Zhao, "New gas sensor head without gas chamber by hollow core PBF," Proc. SPIE 6724, 672407 (2007).

7. A. M. Cubillas, M. Silva-Lopez, J. M. Lazaro, O. M. Conde, M. N. Petrovich, and J. M. Lopez-Higuera, "Methane detection at $1670 \mathrm{~nm}$ band using a hollow-core photonic bandgap fiber and a multiline algorithm," Opt. Express 15, 17570-17576 (2007).

8. A. M. Cubillas, J. M. Lazaro, M. Silva-Lopez, O. M. Conde, M. N. Petrovich, and J. M. Lopez-Higuera, "Methane sensing at $1300 \mathrm{~nm}$ band with hollow-core photonic bandgap fibre as gas cell," Electron. Lett. 44, 403-405 (2008).

9. L. Kornaszewski, N. Gayraud, J. M. Stone, W. N. MacPherson, A. K. George, J. C. Knight, D. P. Hand, and D. T. Reid, "Midinfrared methane detection in a photonic bandgap fiber using a broadband optical parametric oscillator," Opt. Express 15, 11219-11224 (2007).

10. J. Henningsen and J. Hald, "Dynamics of gas flow in hollow core photonic bandgap fibers,” Appl. Opt. 47, 2790-2797 (2008).

11. C. Hensley, D. H. Broaddus, C. B. Schaffer, and A. L. Gaeta, "Photonic band-gap fiber gas cell fabricated using femtosecond micromachining," Opt. Express 15, 6690-6695 (2007).

12. J. P. Parry, B. C. Griffiths, N. Gayraud, E. D. McNaghten, A. M. Parkes, W. N. MacPherson, and D. P. Hand, "Towards practical gas sensing with micro-structured fibres," Meas. Sci. Technol. 20, 075301 (2009).

13. G. Casa, A. Castrillo, G. Galzerano, R. Wehr, A. Merlone, D. Di Serafino, P.Laporta, and L. Gianfrani, "Primary gas thermometry by means oflaser-absorption spectroscopy: determination of the Boltzmann constant," Phys. Rev. Lett. 100, 200801 (2008).

14. C. Daussy, M. Guinet, A. Amy-Klein, K. Djerroud, Y. Hermier, S. Briaudeau, Ch. J. Bordé, and C. Chardonnet, "Direct determination of the Boltzmann constant by an optical method," Phys. Rev. Lett. 98, 250801 (2007).

15. P. Balling, M. Fischer, P. Kubina, and R. Holzwarth, "Absolute frequency measurement of wavelength standard at $1542 \mathrm{~nm}$ : acetylene stabilized DFB laser," Opt. Express 13, 9196-9201 (2005).

16. U. Schünemann, H. Engler, R. Grimm, M. Weidemüller, and M. Zielonkowskic, "Simple scheme for tunable frequency offset locking of two lasers," Rev. Sci. Instrum. 70, 242-243 (1999).

17. http://www.nktphotonics.com.

18. R. Guenther, Modern Optics (Wiley, 1990).

19. R. Amezcua-Correra, F. Gérôme, S. G. Leon-Saval, N. G. R. Broderick, T. A. Birks, and J. C. Knight, "Control of surface modes in low loss hollow-core photonic bandgap fibers," Opt. Express 16, 1142-1150 (2008).

20. D. W. Allan, "Statistics of atomic frequency standards," Proc. IEEE 54, 221-230 (1966).

21. J. Levine, "Introduction to time and frequency metrology," Rev. Sci. Instrum. 70, 2567-2596 (1999).

22. P. Werle, R. Mucke, and F. Slemr, "The limits of signal averaging in atmospheric trace-gas monitoring by tunable diodelaser absorption spectroscopy (TDLAS)," Appl. Phys. B 57, 131-139 (1993).

23. H. Huang and K. Lehman, "Long-term stability in continuous wave cavity ringdown spectroscopy experiments," Appl. Opt. 49, 1378-1387 (2010).

24. E. Li, X. Wang, and C. Zhang, "Fiber-optic temperature sensor based on interference of selective higher-order modes," Appl. Phys. Lett. 89, 091119 (2006). 